\title{
Implementation of Image Registration Techniques and its Applications in Medical Image Analysis
}

\author{
Sugandha Agarwal ${ }^{\# 1}$, O.P. Singh ${ }^{\# 2}$, Deepak Nagaria ${ }^{* 3}$ \\ \#Amity School of Engineering and Technology, Amity University, Lucknow \\ sugandhaa7@gmail.com ${ }^{1}$ \\ *Electronics and Communication Department, BIET, Jhansi
}

\begin{abstract}
This paper aims to classify and analyze recent as well as classic image registration techniques. Image registration is the process of super imposing images of the same scene taken at different times, location and by different sensors. It is a key enabling technology in medical image analysis for integrating and analyzing information from various modalities. Basically image registration finds temporal correspondences between the set of images and uses transformation model to infer features from these correspondences.The approaches for image registration can beclassified according to their nature vizarea-based and feature-based and dimensionalityvizspatial domain and frequency domain. The procedure of image registration by intensity based model, spatial domain transform, Rigid transform and Non rigid transform based on the above mentioned classification has been performed and the eminence of image is measured by the three quality parameters such as SNR, PSNR and MSE. The techniques have been implemented and inferred thatthe non-rigid transform exhibit higher perceptual quality and offer visually sharper image than other techniques.Problematic issues of image registration techniques and outlook for the future research are discussed. This work may be one of the comprehensive reference sources for the researchers involved in image registration.
\end{abstract}

Keywords:MSE (Mean squared error), SNR (Signal to noise ratio), PSNR (Peak signal to noise ratio), MRI (Magnetic Resonance Imaging) CT (Computed Tomography), and PET (Positron Emission Tomography).

\section{INTRODUCTION}

One of the most essential image processing applications of geometric transformation is image registration. Registration is a general term used to describe the process of developing a spatial mapping between sets of data. A technique of image registration allows structural and functional images to be viewed and analyzed in the same coordinate system. It looks for alignment of two or more images of the same scene or it may be images taken at different times. Image registration is a cardinal technology in medical image analysis that has benefited from many years of development. The research in image registration has long history and is indicated in early 1960's to align 3D images. Importance of research and the potential for image registration techniques are identified in early 1980 's. The development of image registration techniques and algorithms is highly complex because it is required to find spatial correspondences among images, and have tremendous applications in Computer Vision, Medical Imaging, Biological Imaging, Brain Mapping, Remote Sensing, Military and Satellite communication etc.

Image registration techniques arerequired to compare data and the images obtained from different measurementsystems based on their applications.Medical images registrations are being used within healthcare for diagnosis, treatmentplanning and monitoring disease progression. Within medical research they are used to ensure disease processes and understand normal improvement and ageing. In various studies, many images are acquired from subjects at different times, and repeatedly with different imaging modalities.

Mostly in the medical imaging field the tasks of fusing of the complimentary and synergistic information acquire by the various imaging modalities are performed. This process is known as multimodal registration. Another common task is the registration of images of the same patient taken at different times and/or in different positions. This process is referred to as mono-modal registration and can be used to track any pathological development. Other applications include inter-patient registration and patient-atlas matching. The previous two applications are generally solved with rigid registrations, in which only rotations and translations are used in the transformation. However the last two applications are generally performed with a non-rigid registration. In this, one image is deformed to match another in order to account for the non-linear local anatomic variations that exist between the images.

The earlier work in medical image registration was registering brain images of the same subject acquired with different modalities (e.g. MRI and CT or PET). Clearly most of the human body does not conform to a rigid or even an affinecalculation and the most interesting and challenging work in registration today involves the development of non-rigid registration techniques for the applications such as correcting the soft-tissue 
deformation during imaging or surgery. Rigid and affine registrations can typically be find in seconds or minutes but most non-rigid registration algorithms require minutes or hours that are being spent in either identifying a geometric set of equivalent features to match directly, or automatically finding a large number of parameters by matching pixel intensities directly. Another concern is that the transformation is asymmetric although there will be a vector that at the scale of the transformation describes how to move each point in the source image to find the corresponding location in the target image, there is no assurance that, at the same scale, every point in the target image can be connected to a corresponding position in the source image.

\section{IMAGE REGISTRATION ALGORITHMS CLASSIFICATION}

\section{A. Intensity-based and feature-based models}

Image registration process can be categories into techniques namely intensity-based and feature based. In this process, one of the images is referred as the reference or the source, and the other image is referred as the target or subject image. The image registration is obtained through spatially registering the target image to align with the reference images. The intensity-based methods compared intensity patterns in image and in feature-based methods; it finds the correspondence between image features such as lines, points and contours. Intensity-based methods registered the entire images and the respective sub-images. If the sub-images are registered then thecenters of corresponding sub images are treated as related feature points. In the Feature-based methods, it establishes a correspondence between a numbers of distinct points in images. After knowing the correspondence points in image, a geometrical transformation is determined to map the target image according to the reference images, which indicates that point-by-point communication is established between the reference and target images.

\section{B. Spatial frequency domain models}

Spatial methods operate in the frequency domain for corresponding intensity features of images patterns. The algorithms developed to find the spatial transformation parameters between source and target images in the image registration process falls into spatial frequency domain models. These models use frequency transformations such as, rotation, translation and scaling in frequency domain. The phase correlation process is used to a pair of images in order to produce a third image which contains a single peak in the frequency domain. The location of this peak corresponds to the relative translation between source and target images.

\section{Non Rigid Registration Method}

Suppose ' $g$ 'signify an observed distorted image and 'R'signify a reference image without turbulent deformation (which can be obtained by averaging the frame sequence) [8]. A non-rigid deformation model can be employed to describe the geometric distortions between these two images. In this model a complex motion is represented by the movements of $n$ control points whose initial position Xoi $=(\hat{x} o i, \hat{y} o i)^{T}(\mathrm{i}=1 \ldots \mathrm{n})$ are equally spaced on the given image ' $g$ '.

The displacement of all the control points on the reference image ' $R$ ' is denoted as the deformation vector:

$$
\overrightarrow{\mathrm{P}}=[\Delta \hat{x} 1, \ldots \ldots \ldots . \Delta \hat{x} n, \Delta \hat{y} 1, \ldots \ldots \ldots . . . \hat{y} n]^{T}
$$

Where $\Delta \hat{\mathrm{x}}, \Delta \hat{\mathrm{y}}$ are the horizontal and vertical displacement from the initial position. The deformed position of any given pixel located at $\mathrm{X}=(x, y)^{T}$ from image $\mathrm{G}$ can then be described as:

$$
\mathrm{W}(\mathrm{X} ; \vec{P})=\mathrm{X}+\mathrm{A}(\mathrm{X}) \vec{P}
$$

Where $\mathrm{A}(\mathrm{X})$ denotes the basis function matrix for $\mathrm{X}$ :

$$
A(X)=\left[\begin{array}{cccc}
c 1 \ldots \ldots . c n & 0 \ldots \ldots .0 \\
0 \ldots \ldots .0 & c 1 \ldots \ldots c n
\end{array}\right]
$$

And $\mathrm{A}(\mathrm{X}) \mathrm{P}$ is the motion vector, which is a linear combination of the movements of all control points. The weight or spline basis $\mathrm{c}_{\mathrm{t}}$ is determined by the distance between $\mathrm{X}$ and $\hat{X}_{o i}$ using B-spline interpolation:

$$
\beta(t)=\left\{\begin{array}{cc}
C_{t}=\beta\left(\frac{\mathrm{x}-\hat{\mathrm{x} o i}}{\mathrm{hx}}\right) \beta\left(\frac{\mathrm{y}-\hat{\mathrm{y} o i}}{\mathrm{hy}}\right) \\
\frac{2}{3}-\left(1-\frac{|\mathrm{t}|}{2}\right) \mathrm{t} 2, \text { if } 0 \leq|\mathrm{t}| \leq 1 \\
\frac{(2-|\mathrm{t}|) 3}{6} & , \quad \text { if } 1 \leq|\mathrm{t}|<2 \\
0 & , \text { otherwise }
\end{array}\right\}
$$

Where $\mathrm{h}_{\mathrm{x}}, \mathrm{h}_{\mathrm{y}}$ are the horizontal and vertical distance between neighboring control points.

In the classic B-spline based registration approach, the deformation vector can be estimated by minimizing the following cost function:

$$
\mathrm{C}(\vec{P})=\sum_{x}|R(W(X ; \vec{P}))-G(X)|^{2}
$$


However, because the above function lacks prior constraint, the resulting estimate is unstable and gets easily stuck in local minima. To improve this method, a stabilization constraint is proposed [2][9][10], which makes the estimated deformation parameters remain small in the regions that have less texture (low image gradient). However, we know that the deformation caused by atmospheric turbulence is independent from image content. In other words, such stabilization constraint would bring unnecessary bias into the estimation.

To accurately estimate the deformation vector and the motion vectors from image R to $\mathrm{G}$, we instead introduce a symmetry constraint into the B-spline registration algorithm. This constraint is based on an important property that the registration should be symmetric or inverse consistent. Let $\mathrm{P}$ denote the Deformation vector that can transform R into $\mathrm{G}$ (forward deformation), and $\mathrm{P}$ denote the inverse vector that transform $\mathrm{G}$ into $\mathrm{R}$ (backward deformation). Thenapproximatelywe have: Combing the two sets of vectors into one vector:

$$
\vec{P}=\overrightarrow{-P} \vec{P}^{T}=\left[\vec{P}^{T}, \vec{P}^{T}\right]
$$

The proposed cost function to minimize becomes

$$
\mathrm{C}(\mathrm{p})=\sum_{x}|R(W(X ; \vec{P})-G(X))|^{2}+\sum_{x}|G(W(X ; \mathrm{P})-R(X))|^{2}+\gamma(\mathrm{P}+\mathrm{P})^{\mathrm{T}}
$$

Where $\gamma$ (scalar) controls the effect of symmetry constraint.

$$
\begin{aligned}
& P^{l+1}=P^{l}-H^{-1} \mathrm{~b} \\
& \mathrm{H}=\sum_{x} d(X) d(X)^{\mathrm{T}}, \mathrm{H}=\sum_{x} d(X) d(X)^{\mathrm{T}} \\
& H=\left[\begin{array}{cc}
\mathrm{H}+\gamma \mathrm{I} & \gamma \mathrm{I} \\
\gamma \mathrm{I} & \mathrm{H}+\gamma \mathrm{I}
\end{array}\right] \\
& d(X)^{T}=\frac{\partial \mathrm{R}(\mathrm{W}(\mathrm{X} ; \mathrm{Pl}))}{\partial \mathrm{W}}, \quad d(X)^{T}=\frac{\partial \mathrm{G}(\mathrm{W}(\mathrm{X} ; \mathrm{Pl}))}{\partial \mathrm{W}} \\
& \mathrm{b}=\sum_{x} d(X)[R(W(X ; \vec{P} \mathrm{l}))-G(X)], \sum_{x} d(X)[G(W(X ; \vec{P} \mathrm{l}))-R(X)]
\end{aligned}
$$

In the above process, the computational cost to calculate the matrix $\mathrm{H}$ is tremendous if it is implemented directly[11].

\section{Rigid/Affine Registration}

In the rigid models, the images are assumed to be of objects that simply need to be rotated and translated w.r.to one another to achieve the required correspondence. Rigid-body transformations consist of only rotations and translations of the image, without changing the preexisting arrangement. It is also termed as linear transformation which includes rotation, scaling, translation and other affine transforms.For each point $(\mathrm{x} 1, \mathrm{x} 2$, $\mathrm{x} 3$ ) in an image, an affine mapping can be defined into the co-ordinates of another space (y1, y2, y3). This is expressed as

$$
\begin{gathered}
y 1=\mathrm{m} 11 \mathrm{x} 1+\mathrm{m} 12 \mathrm{x} 2+\mathrm{m} 13 \times 3+\mathrm{m} 14 \\
y 2=\mathrm{m} 21 \mathrm{x} 1+\mathrm{m} 22 \mathrm{x} 2+\mathrm{m} 23 \times 3+\mathrm{m} 24 \\
y 3=\mathrm{m} 31 \mathrm{x} 1+\mathrm{m} 32 \mathrm{x} 2+\mathrm{m} 33 \times 3+\mathrm{m} 34 y=M_{x}
\end{gathered}
$$

Which is often represented by a simple matrix multiplication $\left(y=M_{x}\right)$ :

$$
\left[\begin{array}{c}
y_{1} \\
y_{2} \\
y_{3} \\
1
\end{array}\right]=\left[\begin{array}{cccc}
m_{11} & m_{12} & m_{13} & m_{14} \\
m_{21} & m_{22} & m_{23} & m_{24} \\
m_{31} & m_{32} & m_{33} & m_{34} \\
0 & 0 & 0 & 1
\end{array}\right]\left[\begin{array}{c}
x_{1} \\
x_{2} \\
x_{3} \\
1
\end{array}\right]
$$

$\mathrm{R}$ is a $3 \mathrm{X} 3$ rotation matrix defined by

$$
\begin{array}{r}
R=r^{\left.(1)_{i j} r^{(2)}\right)_{k}} r^{(3)_{k l}} \\
r^{(1)}=\left[\begin{array}{ccc}
1 & 0 & 0 \\
0 & \cos \alpha & -\sin \alpha \\
0 & \sin \alpha & \cos \alpha
\end{array}\right] \\
r^{(2)}=\left[\begin{array}{ccc}
\cos \alpha & 0 & -\sin \alpha \\
0 & 1 & 0 \\
\sin \alpha & 0 & \cos \alpha
\end{array}\right] \\
r^{(3)}=\left[\begin{array}{ccc}
\cos \alpha & -\sin \alpha & 0 \\
\sin \alpha & \cos \alpha & 0 \\
0 & 0 & 1
\end{array}\right]
\end{array}
$$

The style of formulating these transformations in terms of matrices allows several of them to be combined, by simply multiplying the matrices jointly to form a single matrix. That means repeated re-sampling of data can be avoided as re-orienting an image. Inverse of the affine transformations are obtained by simply inverting the transformation matrix. Rigid registration is achieved by minimization of the sum of squared intensity differences (SSD) between two images. Whenever several images of the similar subject have been acquired, it is extremely 
helpful to have them all register. Some of the easy benefits of this includes, images to be averaged in order to raise signal to noise, or to subtract one image from another to emphasize differences between the images.

\section{E. Extrinsic and Intrinsic registration methods}

Image based registration can also be divided into extrinsic and intrinsic method, in which extrinsic is based on foreign objects introduced into the imaged space whereas intrinsic methods is based on the image information as generated by the patient. Extrinsic methods rely on artificial objects attached to the patient, objects which are designed to be well visible and accurately detectable in all of the pertinent modalities. Intrinsic methods rely on patient generated image content only. Registration can be based on a limited set of identified salient points (landmarks)[17], on the alignment of segmented binary structures (segmentation based), most commonly object surfaces, or directly onto measures computed from the image grey values. Since extrinsic methods by definition cannot include patient related image information, the nature of the registration transformation is often restricted to be rigid translations and rotations only [15][16].

\section{COMPONENTS OF REGISTRATION ALGORITHMS}

A registration algorithm can be divided into three mechanisms viz.Similarity measure, transformation model and Optimization.

\section{A. Similarity measures}

The registration based on patient image content can be divided into geometric approaches and intensity approaches. Geometric approaches construct explicit models of identifiable anatomical elements in all images. These elements normallycontain functionally essential surfaces, curves and point landmarks that can be matched with the second image. These are correspondencewhich define the transformation from one image to the other[12]. The use of such information ensures that the mapping has biological validity and allows the transformation to be interpreted in terms of the fundamental anatomy or physiology[13]. Intensity approaches compare intensity patterns in each image by using mathematical or statistical criteria. They define a measure of intensity comparison between the source and the target image and adjust the transformation until the similarity measure is maximize. They suppose that the images will be mainly similar at the correct registration. Intensity based registrations, match intensity pattern over the whole image but do not utilize anatomical knowledge. Geometric registration use anatomical information but usually sparsely distributed throughout the images. Combination of both geometric features and intensity features in registration should result in more robust methods.

B. Transformation models

The transformation model defines how one image can be deformed to match another; it characterizes the type and number of possible deformations. These parameters are applied to a vector locating a point in an image to find its location in another image. Transformation model serve two purposes firstof all it controls how image features can be moved relative to one another to improve the image similarity and second it interpolates between those features where there is no important information[14]. The most well-known example is rigid or affine transformation that can be described very compactly. Transformations used in non-rigid registration range from smooth regional difference described by a small number of parameters. One of the most important transformations is the family of splines that have been use in various forms for around 15 years. Spline-based registration algorithms use corresponding control points, in the source and target image and a spline function to define correspondences away from these points.

C. Optimization

Optimization refers to the manner in which the transformation is adjustedto improve the image similarity. A good optimizer is one that reliably and quickly determines the best possible transformation. Choosing a good optimizer requires a good knowledge of the registration problem, the constraints that can be applied and knowledge of numerical analysis. For the optimizer this means that more time is required to make a parameter choice and that there is more chance of choosing a set of parameters, which result in a good image match which is yet not the best one.

\section{RESULT AND DISCUSSION}

Registration plays a vital role in the medical imaging field where persistent advances in imaging modalities, including MRI, CTI and PET, allow the generation of 3D images that explicitly outline detailed in vivo information of not only human anatomy, but also human function. For implementing image registration algorithms like Intensity based model, spatial domain transform, Rigid transform and Non Rigid transform we have incorporated MRI of brain image. The registration geometrically aligns two images viz reference and target images A slight distorted image known as target image matches with the reference image by the means of the algorithms described above such as intensity based and feature based, spatial transformation, rigid and non-rigid transformation.And the parameters like Signal to Noise Ratio (SNR), Peak Signal to Noise Ratio (PSNR) and Mean Square Error (MSE) are calculated for all the above techniques to assess the MRI image quality with 
Gaussian noise factor 0.11. By analyzing the results we infer thatNon rigid transformation has high SNR and PSNR comparing with other techniques. But the MSE of rigid transform is less than non-rigid transform. Hence for better image quality and smoothen edges we prefer non rigid transform but when we require a reduced amount of error in the content of image and validate results we choose rigid transform.

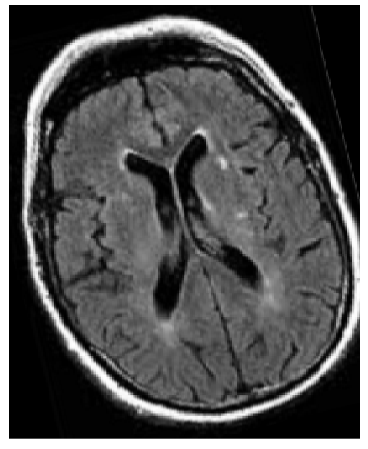

(1a)Reference Image

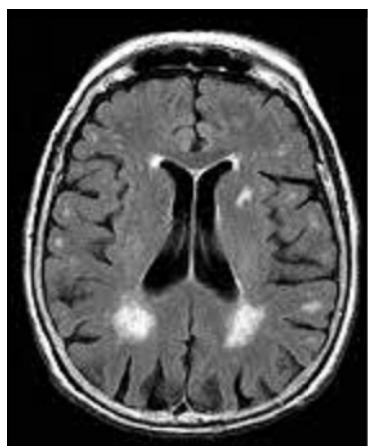

(1b)Target Image

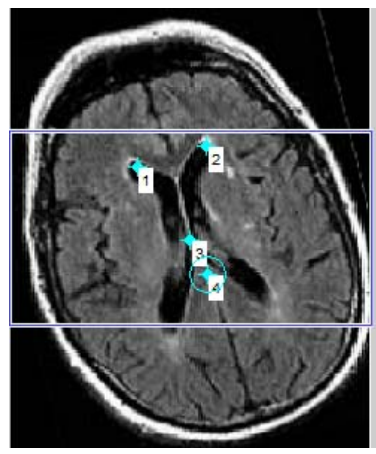

(1c)Points of Registration

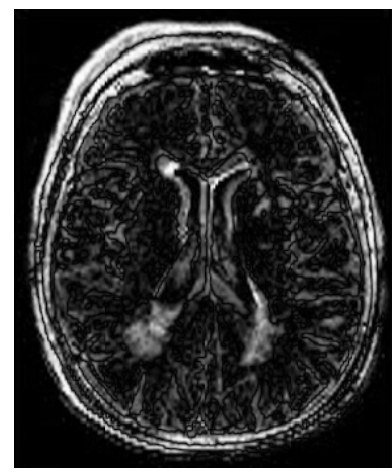

(1f) Rigid Registration

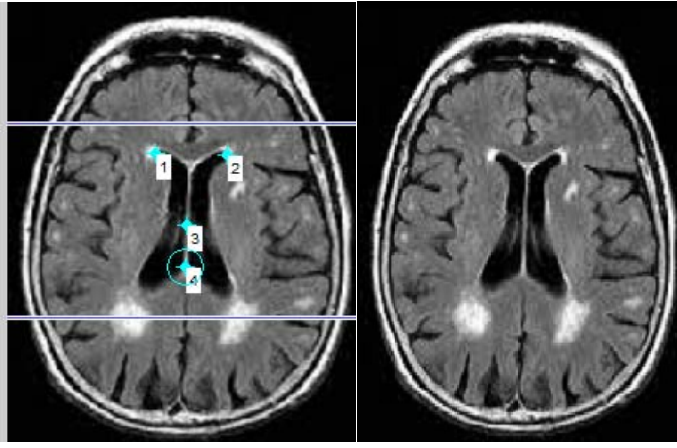

(1d) Intensity based transform

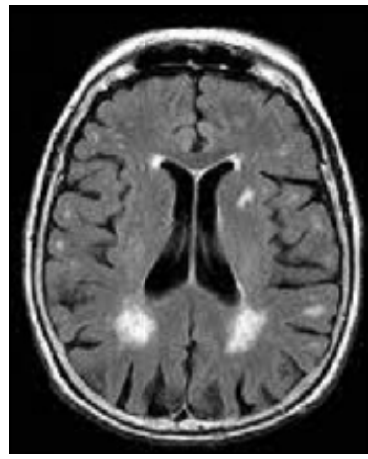

(1g) Non Rigid Registration

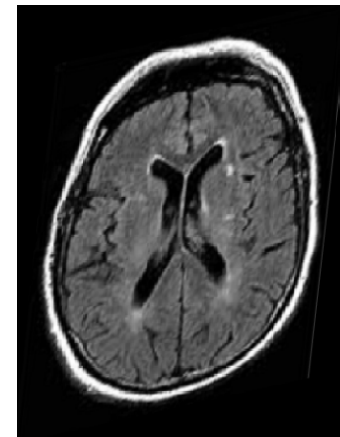

(1e) Spatial Transform

Figure 1: Implication of Various Image Registration Techniques

Table I. Tabular Representation of SNR, PSNR, and MSE of Different Images

\begin{tabular}{|l|r|r|r|}
\hline Techniques & \multicolumn{1}{|c|}{ SNR } & \multicolumn{1}{c|}{ PSNR } & \multicolumn{1}{l|}{ MSE } \\
\hline Original image & 12.4484 & 17.6495 & 65.4949 \\
\hline Target image & 11.9129 & 33.0640 & 60.8306 \\
\hline $\begin{array}{l}\text { Intensity based } \\
\text { transform }\end{array}$ & 12.5752 & 33.0821 & 59.9489 \\
\hline $\begin{array}{l}\text { Spatial domain } \\
\text { transform }\end{array}$ & 12.6882 & 33.1401 & 64.6990 \\
\hline Rigid transform & 13.3548 & 33.2040 & 49.3258 \\
\hline Non rigid registration & 14.2693 & 33.6176 & 62.6433 \\
\hline
\end{tabular}




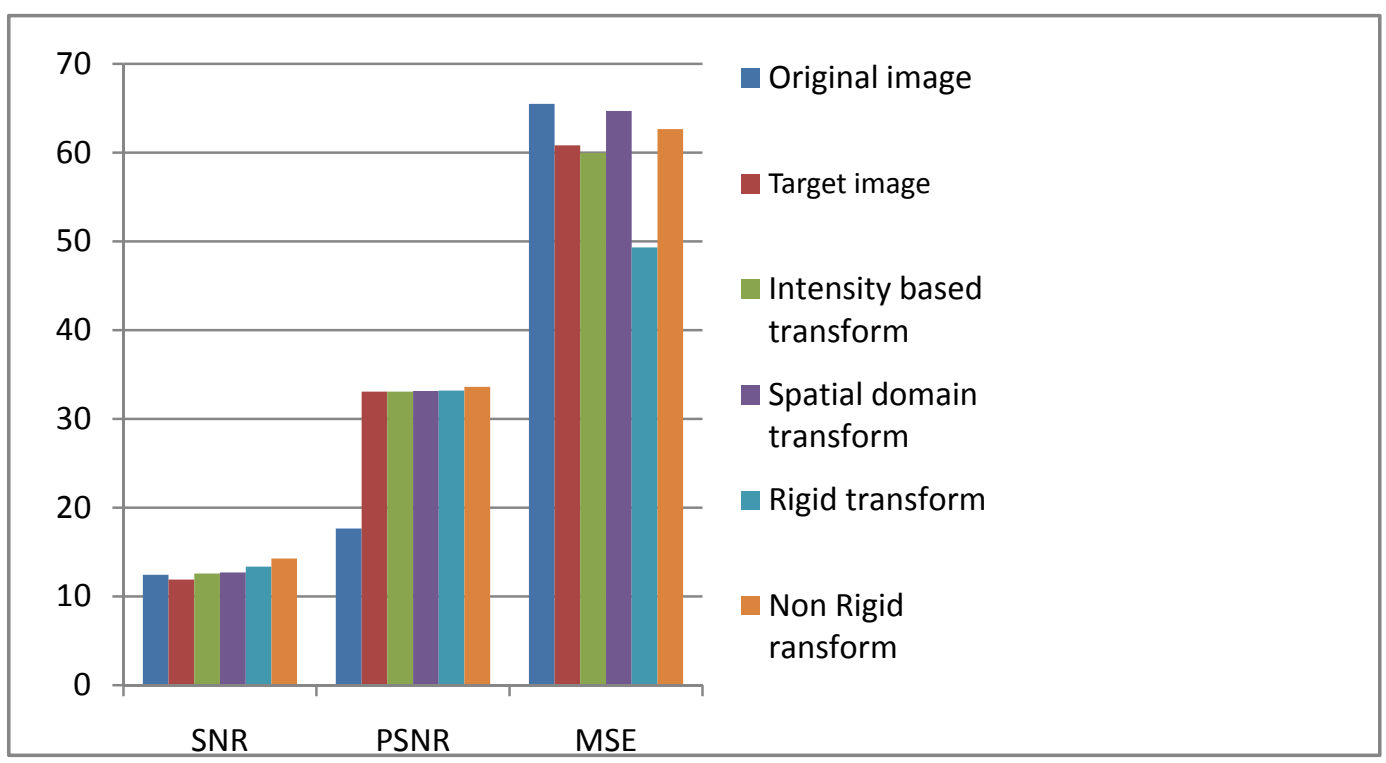

Fig. 2.Graphical Representation of various image registration techniques

\section{CONCLUSION}

In this work, we have presented various methods to acquire image registration classified by the nature and dimensionalities. By implementing and analyzing the above mentioned image registration algorithm, it was found that non rigid transform technique out performs the rigid transform, spatial domain transform and intensity based transform in terms of SNR and denoised image appear visually more pleasant than the other methods. The non-rigid transform provides high SNR values and can remove Gaussian noise of 0.11 from MRI images very competently, but doesn't effectively improve MSE. Hence non rigid transform is not suited for reducing error though it recovers the edges perfectly. For images, it is important that edges data should be preserved. The denoising algorithm presented in this work, to a large extent has satisfied the constraint that phase should not be corrupted. The effectiveness of this technique encourages the possibility of improving this approach to preserve the edges to more extent. Image registration plays a very dominant role in panoramic image generation and creation, in which the utility are incomprehensible for researchers in any field of application.

\section{REFERENCES}

[1] Vani.M, Shruthi.M.K "Removing Atmospheric Turbulence Using Restoration Algorithm" e-ISSN: 2278-0661, p- ISSN: 22788727Volume 16, Issue 4, Ver. I (Jul-Aug. 2014).

[2] Shimizu, M., Yoshimura, S., Tanaka, M., and Okutomi, M., "Super-resolution from image sequence under influence of hot-air optical turbulence," CVPR 2008, 1-8 (June 2008).

[3] SindhuMadhuriG "Classification of Image Registration Techniques and Algorithms in Digital Image Processing - A Research Survey" International Journal of Computer Trends and Technology (IJCTT) - volume 15 number 2 - Sep 2014.

[4] Medha V. Wyawahare, Dr. Pradeep M. Patil, and Hemant K. Abhyankar "Image Registration Techniques: An overview" International Journal of Signal Processing, Image Processing and Pattern Recognition Vol. 2, No.3, September 2009.

[5] J. B. Antoine Maintz1 and Max A. Viergever "An Overview of Medical Image Registration Methods",American Journal of Biomedical Engineering 2016, 6(2): 53-58.

[6] Dieter A. Hahn, Volker Daum, and Joachim Hornegger “Automatic Parameter Selection for Multimodal Image Registration" IEEE transactions on medical imaging, vol. 29, no. 5, may 2010.

[7] Xiang Zhu, Student Member "Removing Atmospheric Turbulence via Space-Invariant Deconvolution" IEEE transactions on pattern analysis and machine intelligence, vol. 35, no. 1, January 2013.

[8] M. Shimizu, S. Yoshimura, M. Tanaka, and M. Okutomi, "SuperResolution from Image Sequence under Influence of Hot-Air Optical Turbulence," Proc. IEEE Conf. Computer Vision and Pattern Recognition, June 2008.

[9] S. Farsiu, M. Elad, and P. Milanfar, "Constrained, Globally Optimal, Multi-Frame Motion Estimation," Proc. IEEE/SP 13th Workshop Statistical Signal Processing, pp. 1396-1401, July 2005.

[10] M.F. Beg and A. Khan, "Symmetric Data Attachment Terms for Large Deformation Image Registration," IEEE Trans. Medical Imaging, vol. 26, no. 9, pp. 1179-1189, Sept. 2007.

[11] X. Zhu and P. Milanfar, "Image Reconstruction from Videos Distorted by Atmospheric Turbulence," Proc. SPIE Electronic Imaging Conf. Visual Information Processing and Comm., Jan. 2010.

[12] Rohr K., Dordrecht , "Landmark-based image analysis: using geometric and intensity models," Computational Imaging and Vision Series., Boston, Kluwer Academic Publishers, Volume $21,2001$.

[13] Rohr K, Stiehl HS, Sprengel R, Beil W, Buzug TM, Weese J, et al.,"Point-based elastic registration of medical image data using approximating thin-plate splines," Visualization in Biomedical Computing, 1996; 1131:297-306.

[14] Woods RP, Grafton ST, Watson JDG, Sicotte NL, Mazziotta JC, "Automated image registration: II. Intersubject validation of linear and nonlinear models" J Comput Assist Tomogr 1998; 22:153-65.

[15] D. A. Simon, R. V. O’Toole, M. Blackwell, F. Morgan, A. M. DiGioia, and T. Kanade., "Accuracy validation in image-guided orthopaedic surgery,'InMedicalrobotics and computer assisted surgery, pages 185-192. Wiley, 1995. 
[16] R. E. Ellis, S. Toksvig-Larsen, M. Marcacci, D. Caramella, and M. Fadda.,"A biocompatible fiducial marker for evaluating the accuracy of CT image registration,"international congress series, pages 693-698, Amsterdam, 1996. Elsevier.

[17] A.C. Evans, S. Marrett, J. Torrescorzo, S. Ku, and L. Collins., "MRI-PET correlation in three dimensions using a volume of interest (VOI) atlas,'Journal of cerebral blood flow and metabolism, 11:A69-A78, 1991.

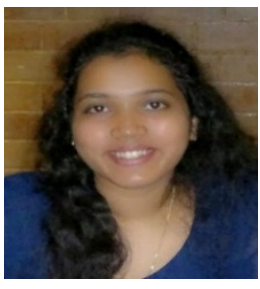

Sugandha Agarwal was born in 1987. She completed her B.Tech (ECE) in 2009 from Uttar Pradesh technical university and M.Tech (ECE) in 2012 from Amity University. She is currently working as assistant professor in electronics and communication engineering department, Amity University Uttar Pradesh Lucknow Campus. She is also a member of Institution of Engineering \& Technology (IET-UK) and Institute of Electrical and Electronics Engineers (IEEE). Her area of research include digital image processing, networking, digital communication, etc. email id: sugandhaa7@gmail.com

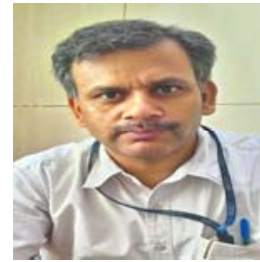

Professor O.P Singh has completed his Ph.D. degree from IIT BHU. He had a work experience of sixteen years in teaching. Presently he is head of department of electrical and electronics in Amity School of Engineering \& Technology, Amity University Uttar Pradesh Lucknow Campus. He is also a member of Indian society of remote sensing (ISRS), the Institution of Engineering \& Technology (IET-UK) and Material Research Society of India (MRSI). His area of research include digital electronics engineering, microwave and antenna design, control system, pattern recognition in image compression etc. Email id- opsing@amity.edu

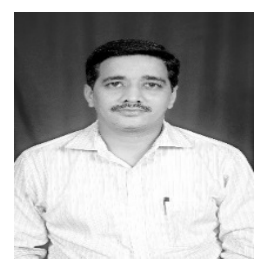

Deepak Nagaria is with BIET, Jhansi, INDIA as Associate Professor in the Department of Electronics \& Communication Engineering. Presently he is Additional Controller of Examination at Dr. APJ Abdul Kalam Technical University, Lucknow. He received his B.Tech from BIET, Jhansi, India in 1996 and master's degree from National Institute of Technology, Kurukshetra, INDIA in 1999. He has completed his Ph. D. from Indian Institute of Technology, Roorkee, INDIA in 2009. He is in teaching profession since 1999. His research interest includes Control Systems, Artificial Intelligence, Signal Processing. He is member of various academic research councils and societies. deepaknagaria@gmail.com. 